\title{
Extensive meningeal and prenchymal calcified tuberculoma as long-term residual sequelae of tuberculous meningitis
}

\author{
Bon D. Ku, Seung Don Yoo ${ }^{1}$ \\ Department of Neurology, Myongji Hospital, 697-24, Hwajung-dong Dukyang-gu Goyang, South Korea, ${ }^{1}$ Department of Physical \\ Medicine and Rehabilitation, Kyung Hee University School of Medicine, Seoul, Korea
}

\begin{abstract}
A 51-year-old woman presented with progressive cognitive decline of 1 year duration. Her past medical history revealed that she received treatment for tuberculous meningitis and milliary tuberculosis at the age of twenty. At that time cerebrospinal fluid (CSF) and sputum were positive for M. tuberculosis. During that illness she had suffered from recurrent seizures. Neurological examination revealed right optic atrophy with total blindness and right spastic hemiparesis. Neuropsychological tests revealed visuospatial, verbal and visual memory deficits, and also deficits in frontal executive functions. Brain computed tomography (CT) scan and magnetic resonance imaging (MRI) showed multiple extensive popcorn-like calcifications in the basal cisterns, insula, hippocampus, and frontal corticomedullary junction along the meninges protruding into the brain prenchyma [Figures 1 and 2].
\end{abstract}

The masses showed hypointensity in all spin-echo sequences of the brain MRI [Figure 2]. These findings suggest extensive meningeal and parenchymal calcified tuberculoma as long-term sequelae of tuberculous meningitis. A large calcified nodule in the right crus cerebri was probably responsible for the blindness in her right eye and a right hippocampal head compression by the calcified lesion may relate to some of her cognitive deficits [Figure 3].

Tuberculosis usually involves the brain through hematogenous spread. Hematogenously disseminated $M$. tuberculosis lodge in the corticomedullary junctions where a rupture into the subarachnoid space leads to meningeal infection and granuloma formation in the base cisterns. ${ }^{[1]}$ These focal granulomas may go through calcific stage. ${ }^{[1,2]}$ Calcified tuberculomas as a sequelae

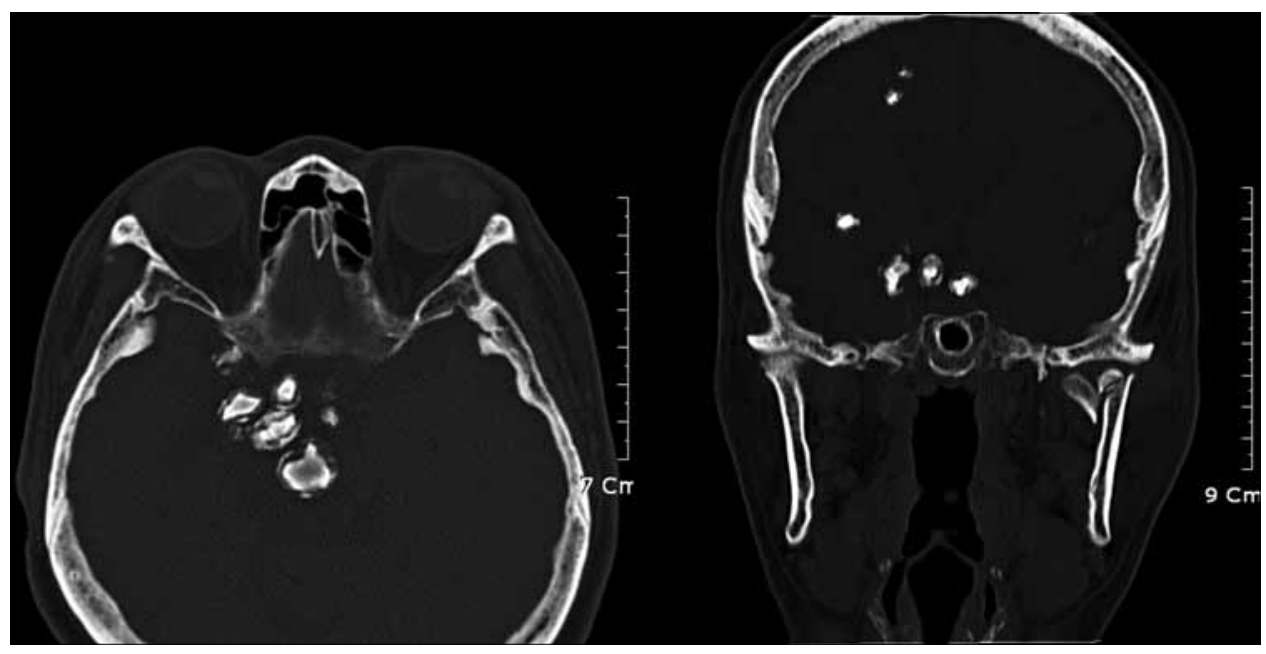

Figure 1: The brain CT showed multiple extensive popcorn-like calcifications in basal cisterns, insular, hippocampus and frontal corticomedullary junction along the meninges protruding into brain parenchyma 


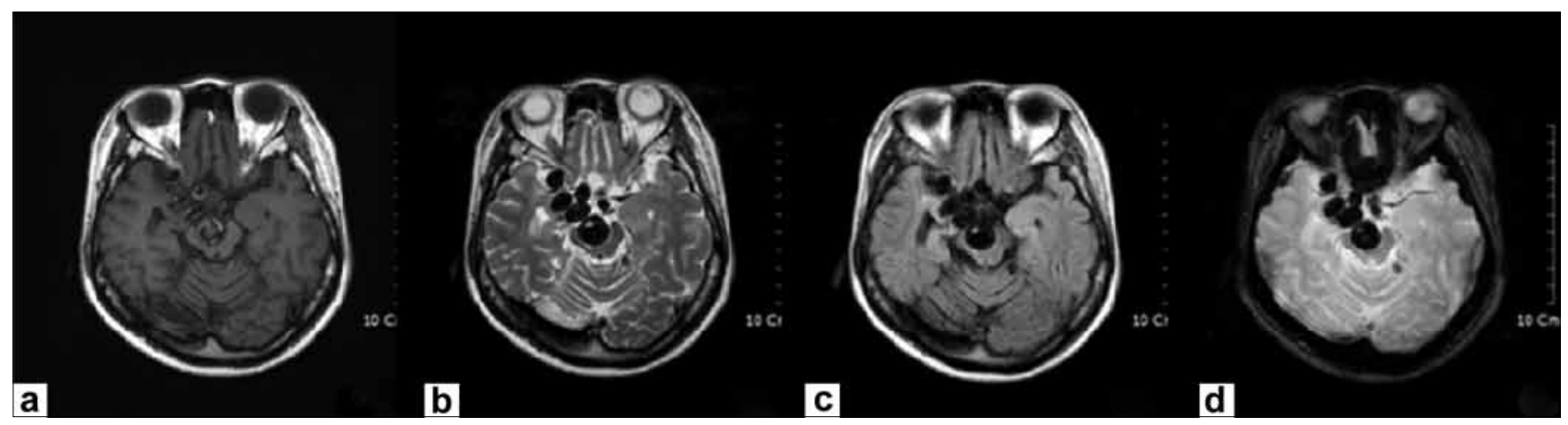

Figure 2: Multiple extensive hypointense calcified tuberculomas in the basal cisterns, insular and frontal corticomedullary junction in the all spin-echo sequences of axial brain MRI (a: T1-weighted axial, b: T2-weighted axial, c: FLAIR axial, d: Gradient echo axial)

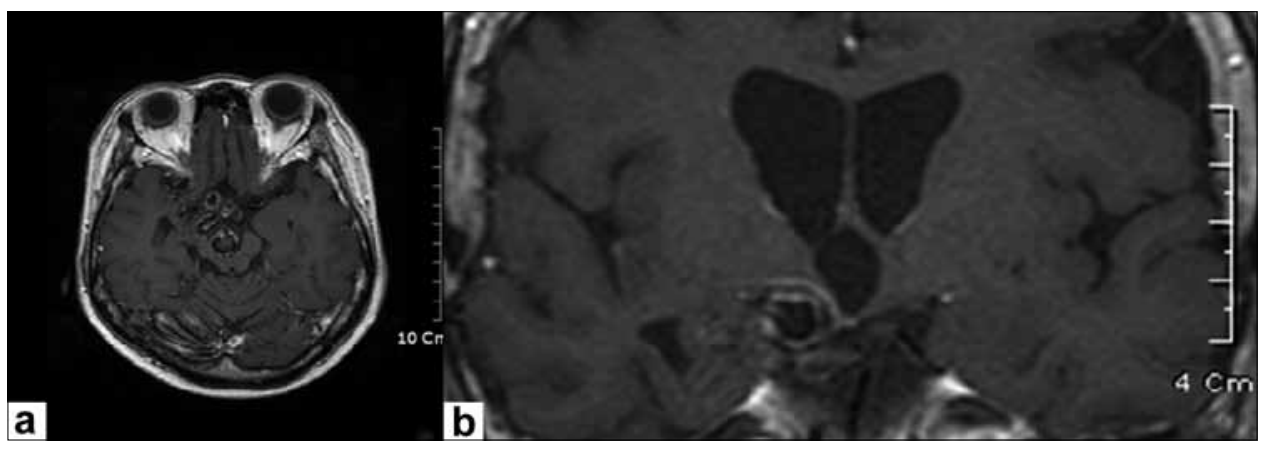

Figure 3: The calcified nodules pressure right side crus cerebri (a) and head of hippocampus (b, arrow) resulting total blindness of right eye and cognitive decline respectively in T1-weighted brain MRI

of meningitis usually appear markedly hypointense on all spin-echo sequences. ${ }^{[2]}$ An isointense or hypointense core with a hyperintense rim on T2-weighted and fluid attenuated inversion recovery (FLAIR) images is the most common presentation. ${ }^{[2]}$ The inhomogeneous ring-like high signal in the T1-weighted image suggests peripheral gliotic changes of calcification.

\section{Address for correspondence:} Prof. Bon D. Ku, Department of Neurology, Myongji Hospital, 697-24, Hwajung-dong Dukyang-gu Goyang, South Korea. E-mail: neurodasan@paran.com DOI: $10.4103 / 0028-3886.55593$

\section{References}

1. Chang KH, Han MH, Roh JK, Kim IO, Han MC, Choi KS, et al. Gd-DTPA enhanced MR imaging in intracranial tuberculosis. Neuroradiology 1990;32:19-25.

2. Wasay M, Kheleani BA, Moolani MK, Zaheer J, Pui M, Hasan S, et al. Brain CT and MRI findings in 100 consecutive patients with intracranial tuberculoma. J Neuroimaging 2003;13:240-7.

Accepted on 09-02-2009

Source of Support: Grant of the Korea Healthcare Technology R\&D Project, Ministry for Health, Welfare \& Family Affairs, Republic of Korea (A050079), Conflict of Interest: None declared. 